Research Paper

\title{
Positive impact of the negative lymph node count on the survival rate of stage III colon cancer with pN1 and right-side disease
}

\author{
Qi Quan1,2\#, Mingshu Zhu1,3\#, Shousheng Liu1,2\# , Ping Chen1,2, Wenzhuo He1,2, Yuanyuan Huang1,2, \\ Yuming Rong ${ }^{1,2}$, Huijuan Qiu ${ }^{1,2}$, Bei Zhang ${ }^{1,2}$, Liangping Xia ${ }^{1,2}$ \\ 1. State Key Laboratory of Oncology in South China, Collaborative Innovation Center for Cancer Medicine, Guangzhou, Guangdong, China. \\ 2. VIP Region, Sun Yat-Sen University Cancer Center, Guangzhou, China. \\ 3. Department of Pathology, Sun Yat-Sen University Cancer Center, Guangzhou, China. \\ \# These authors contributed equally to this work and should be considered co-first authors. \\ $\square$ Corresponding authors: Liangping Xia, PhD; Bei Zhang, MD, VIP Region, Sun Yat-Sen University Cancer Center, 651 Dongfeng East Road, Guangzhou, \\ Guangdong, China 510060. Tel: 86-20-87343454; Fax: 86-2087343454; E-mail: xialiangping@163.com \\ (c) Ivyspring International Publisher. This is an open access article distributed under the terms of the Creative Commons Attribution (CC BY-NC) license \\ (https://creativecommons.org/licenses/by-nc/4.0/). See http://ivyspring.com/terms for full terms and conditions.
}

Received: 2017.11.09; Accepted: 2018.10.24; Published: 2019.01.29

\begin{abstract}
Background: We aimed to investigate the role of the negative lymph node count (NLN) as a predictor of prognosis in patients with stage III colon cancer.

Methods: We conducted a retrospective study on patients who were diagnosed with stage III colon cancer at Sun Yat-sen University Cancer Center. According to the number of negative lymph nodes, all patients were divided into the low or high NLN group. Overall survival (OS) and disease-free survival (DFS) were assessed using the Kaplan-Meier method and log-rank test between the two groups. Univariate and multivariate Cox proportional hazards models were used to evaluate the risk factors for survival.

Results: The time-dependent receiver operating characteristic (ROC) curve showed that the optimal cutoff value of NLN was nine. In total, 167 and 298 patients were distributed into the low and high NLN groups, respectively. Patients in the high NLN group tended to present with a greater proportion of right-side colon cancer and $\mathrm{pN1}$ stage disease, superior DFS $(P<0.001)$ and $O S(P=0.001)$ than those in the low NLN group. Multivariable analyses confirmed increased NLN as a positive prognostic variable, independent of other potential confounding factors. Subgroup analysis showed that in patients with a right-side location, those with 9 or fewer negative lymph nodes had a 5 -year OS rate of $35.4 \%$ versus $77.1 \%$ in those with more than 9 negative lymph nodes evaluated $(\mathrm{P}<0.001)$. For patients with stage $\mathrm{pN} 1$, those with $\mathrm{NLN} \leq 9$ exhibited an inferior 5 -year OS rate than those with NLN $>9(71.1 \%$ vs $84.8 \%$, respectively; $P=0.009)$. There was no association between the number of negative lymph nodes identified and survival for patients with stage pN2 and left-side disease.

Conclusion: NLN is an important prognostic factor for stage III colon cancer patients with right-side and stage $\mathrm{pN} 1$ disease other than for patients with stage pN2 and left-side disease, which can be partly explained in terms of inflammation and immunity.
\end{abstract}

Key words: negative lymph node count; colon cancer; prognosis; survival

\section{Introduction}

Colorectal cancer is the fourth most frequently malignancy in humans. For resectable non-metastatic colon cancer, the preferred surgical approach is colectomy with en bloc removal of the regional lymph nodes [1,2]. Lymph node metastasis is a major indicator of the postoperative staging and prognosis of colorectal cancer [3].

In recent years, some scholars have noted that other parameters related to the status of lymph nodes, such as the number of lymph nodes examined, proportion of positive lymph nodes (lymph node ratio, LNR), number of negative lymph nodes (NLN) 
and positive lymph node logarithmic ratio (LODDS), significantly impact the prognosis of colorectal cancer patients [4-8]. In particular, a higher negative lymph node count has been shown to be correlated with longer overall survival in various tumors, including breast cancer, cervical cancer and gastric cancer, in those who received treatment, such as preoperative radiotherapy [9-11]. In the field of colorectal cancer, in one of the largest studies, Paul MJ statistically analyzed the clinical data of 20,702 patients with colon cancer in the US SEER database and studied the relationship between the number of negative lymph nodes and prognosis of stage III colon cancer. The results of multivariate analysis showed that there was a significant difference in the number of NLNs $\geq 13$ and $<13$ in IIIB and IIIC colon cancer when the number of positive lymph nodes was excluded from the prognosis [7].

The mechanism underlying the correlation between increased NLNs and longer survival is poorly understood. Several hypotheses have been related to patient care, including accurate tumor staging, more efficacious surgical intervention, and superior quality of pathology service. Some investigators have raised the possibility of an underlying biologic mechanism of action, including a greater host immune response to tumors among patients with a larger negative lymph node count. Paul MJ questioned the prevailing dogma that the relationship is simply due to stage migration or quality of care [7].

The large-sample data from Paul MJ confirmed a positive correlation between NLN and the prognosis of stage III colon cancer. However, they did not provide evidence to support or refute the hypothesis that tumor-host interactions are important. In addition, the data were obtained from patients treated between 1988 and 1997; there has been considerable progress in the treatment of colorectal cancer over the past 20 to 30 years, and the understanding of this treatment has expanded to include an awareness of the inflammatory and immune status, as well as differences between right-side and left-side colon cancer. We conducted a retrospective study to assess whether the role of the negative lymph node count in the prognosis of stage III colon cancer is related to other clinicopathological factors associated with the host immune response and inflammation action.

\section{Patients and Methods}

\section{Ethics Statement}

The study complied with the standards of the Declaration of Helsinki and was approved by the Research Ethics Committee at the Cancer Center of
Sun Yat-sen University. Written informed consent was obtained from each patient.

\section{Patient Selection}

This study enrolled patients who were pathologically diagnosed with stage III colon cancer and underwent surgery from March 2006 to December 2014 at the Sun Yat-Sen University Cancer Center in China. Patients were excluded for having insufficient data regarding the tumor location or complete pathology information, including the number of LNs assessed. Patients who received preoperative radiotherapy or chemotherapy were also excluded.

\section{Clinical data collection}

The clinical data contained the following information: age, gender, weight, height, primary tumor site, tumor stage, node stage, tumor histological grade, nerve invasion or vascular invasion status, serum carcinoembryonic antigen (CEA), serum CA199, albumin, C-reactive protein (CRP), neutrophil-to-lymphocyte ratio (NLR), adjuvant chemotherapy regimen, and tumor microenvironment parameters. The tumor microenvironment parameters were assessed as previously reported $[12,13]$. We studied neutrophil and lymphocyte infiltration (in both the central region and invasive margin) and used a four-degree scale to assess infiltration of neutrophils and lymphocytes. A score of 0 indicated an absence of reaction, and a score of 1 indicated a weak increase in each cell type, a score of 2 represented a moderate increase in each cell type, and a score of 3 indicated a severe increase in each cell type. Absent and weak (0-1) were combined as a low tumor grade, while a moderate to severe increase (2-3) was considered a high tumor grade.

There is no uniform marginal value for the number of negative lymph nodes that is associated with tumor outcome; thus, we analyzed the receiver operating characteristic (ROC) curve to provide an optimal critical value for our data.

\section{Patient Follow-Up and Statistical Analysis}

Overall survival (OS) was defined as the interval between the diagnosis date and time of death for any reason or the last follow-up. Disease-free survival (DFS) was defined as the time from the radical surgery to disease relapse or death. Statistical analyses were conducted using SPSS standard version 22.0 (IBM Corporation, Armonk, NY) for Windows. Categorical characteristics were compared by chi-square test. Survival curves were analyzed by the Kaplan-Meier method, and the log-rank test was used to compare differences. 


\section{Results}

\section{Patient Characteristics}

We evaluated a series of 465 pathologically confirmed stage III colon cancer patients who underwent curative surgical resection with regional lymph node dissection at Sun Yat-Sen University Cancer Center. The clinical baseline features of the 465 cases are listed in Table 1 . The median age at diagnosis was 58 years (range, $19-85$ years), and $58.1 \%$ of the patients were male. A total of $65(26.7 \%)$ patients and $104(42.7 \%)$ presented with nerve invasion and vascular invasion. Patients of stage IIIB accounted for $79.8 \%$ of the total number of patients. A minority of the patients $(24.9 \%)$ exhibited increased CA199, and only 23 patients had increased CEA. A total of $177(38.1 \%)$ and $288(61.9 \%)$ patients presented with primary right and left locations, respectively. Adjuvant chemotherapy was administered to 445 $(95.7 \%)$ patients, with most of the patients $(88.0 \%)$ receiving oxaliplatin-containing regimens. Elevated CPR and NLR occurred in 100 (23.5\%) and 230 (49.5\%) patients, respectively. Among all the 243 patients with neutrophil and lymphocyte infiltration, 150 (61.7\%) and $62(25.5 \%)$ patients were found to be high-grade central and invasive neutrophil infiltration, respectively. A total of $92(37.9 \%)$ patients and 68 $(28.0 \%)$ patients displayed high-grade central and invasive lymphocyte infiltration, respectively.

\section{Correlation between the negative lymph node count and Clinical Features}

The optimal cutoff value for the number of negative lymph nodes was nine based on the ROC analysis results (area under the curve: 0.641, 95\% confidence interval [CI]: 0.585-0.697, $\mathrm{P}<0.001$ ) (Figure 1). Correlations between the NLN and clinicopathological factors are summarized in Table 1. Patients in the high NLN group presented with a greater proportion of right-side colon cancer $(47.3 \%$ vs $21.6 \%, \mathrm{P}=0.005)$ and stage $\mathrm{pN} 1(73.5 \%$ vs $60.5 \%, \mathrm{P}$ $<0.001$ ) disease than patients in the low NLN group. Patients' characteristics in the two groups were well balanced for age, gender, $\mathrm{T}$ stage, histological grade, adjuvant chemotherapy regimen, CRP, NLR and neutrophil and lymphocyte infiltration (Table 1).

\section{Survival Analysis}

The cutoff point for follow-up data collection was May 22, 2017. In the current study, the 3-year DFS rate and 5-year OS rate of all patients were $71.1 \%$ and $77.5 \%$, respectively. Compared with patients with NLN $\leq 9$, patients with NLN $>9$ experienced a significantly superior 5 -year OS rate $(82.7 \%$ vs $68.6 \%$, $\mathrm{P}<0.001)$ and 3-year DFS rate $(79.1 \%$ vs $56.8 \%, \mathrm{P}=$ 0.001) (Figure 2) (Table 2).
Table 1. Baseline characteristics of patients by negative LNs.

\begin{tabular}{|c|c|c|c|}
\hline Characteristics & $\mathrm{NLN} \leq 9$ & NLN $>9$ & $\mathrm{p}$-value \\
\hline \multicolumn{4}{|l|}{ No. of cases } \\
\hline Age at diagnosis (years) & & & 0.141 \\
\hline$\leq 60$ & 91 & 184 & \\
\hline$>60$ & 76 & 114 & \\
\hline Gender & & & 0.845 \\
\hline Male & 98 & 172 & \\
\hline Female & 69 & 126 & \\
\hline Primary tumor site & & & $<0.001$ \\
\hline Right & 36 & 141 & \\
\hline Left & 131 & 157 & \\
\hline Tumor stage & & & 0.151 \\
\hline $\mathrm{T} 1-2$ & 17 & 19 & \\
\hline T3-4 & 150 & 279 & \\
\hline Node stage & & & 0.005 \\
\hline $1 \mathrm{a}-1 \mathrm{c}$ & 101 & 219 & \\
\hline $2 a-2 b$ & 66 & 79 & \\
\hline Tumor histological grade & & & 0.754 \\
\hline high or moderate & 113 & 207 & \\
\hline Low or mucin adenoid & 54 & 91 & \\
\hline Nerve invasion ${ }^{\mathrm{a}}$ & & & 0.350 \\
\hline No & 53 & 125 & \\
\hline Yes & 24 & 41 & \\
\hline Vascular invasion ${ }^{\mathrm{a}}$ & & & 0.493 \\
\hline No & 36 & 86 & \\
\hline Yes & 24 & 80 & \\
\hline Stage (7th AJCC) & & & 0.006 \\
\hline IIIa & 18 & 17 & \\
\hline $\mathrm{IIIb}$ & 120 & 251 & \\
\hline IIIc & 29 & 30 & \\
\hline Adjuvant chemotherapy & & & 0.199 \\
\hline No chemotherapy & 10 & 10 & \\
\hline 5-FU alone & 16 & 20 & \\
\hline Oxaliplatin-containing & 141 & 268 & \\
\hline CEA(ng/mL) & & & 0.824 \\
\hline$\leq 5$ & 158 & 284 & \\
\hline$>5$ & 9 & 14 & \\
\hline CA199(ng/mL) & & & 0.739 \\
\hline$\leq 35$ & 127 & 222 & \\
\hline$>35$ & 40 & 76 & \\
\hline $\mathrm{CRPb}$ & & & 0.152 \\
\hline$\leq 8.2$ & 121 & 205 & \\
\hline$>8.2$ & 29 & 71 & \\
\hline NLR & & & 0.334 \\
\hline$\leq 2.06$ & 79 & 156 & \\
\hline$>2.06$ & 88 & 142 & \\
\hline Ne Central Group ${ }^{c}$ & & & 0.777 \\
\hline low & 28 & 65 & \\
\hline high & 49 & 101 & \\
\hline Ne Invasive Group ${ }^{c}$ & & & 0.157 \\
\hline low & 62 & 119 & \\
\hline high & 15 & 47 & \\
\hline Ly Central Groupc & & & 0.258 \\
\hline low & 52 & 99 & \\
\hline high & 25 & 67 & \\
\hline Ly Invasive Groupc & & & 0.094 \\
\hline low & 61 & 114 & \\
\hline high & 16 & 52 & \\
\hline
\end{tabular}

Abbreviations:

LN, lymph node count; CEA, carcino-embryonic antigen; CA199, ca antigen 199; $\mathrm{Ne}$, neutrophil; Ly, lymphocyte; NLR, neutrophil-to-lymphocyte ratio. Note: a Nerve invasion and vascular invasion were recorded in 243 patients. bCRP were recorded in 426 patients.

\# Ne Central, Ne Invasive, Ly Central, Ly Invasive was measured in 243 patients.

In multivariate analyses, as shown in Table 3, the number of negative lymph nodes was an independent prognostic indicator of DFS (hazard ratio [HR]: 0.309, 95\% CI: 0.187 - 0.509, P < 0.001) and OS (HR: $0.20495 \%$ CI: $0.073-0.567, \mathrm{P}=0.002)$. Additionally, invasive lymphocyte infiltration was considered an 


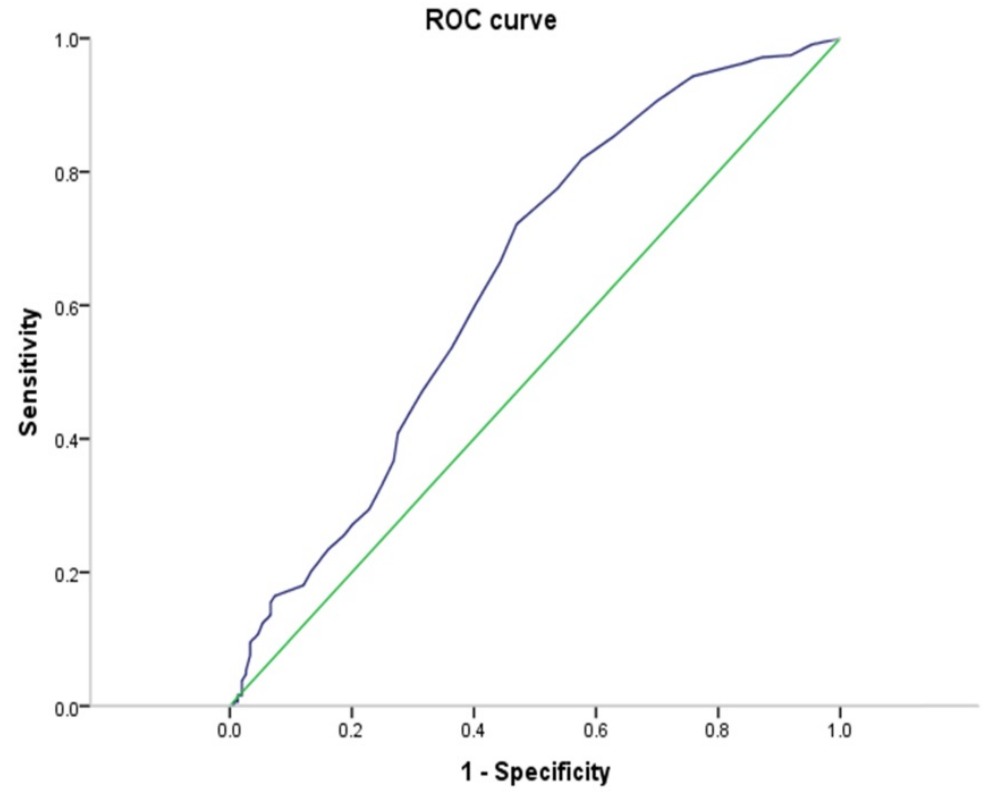

Figure 1. ROC of the negative lymph node counts for DFS prediction. Notes: Area under the ROC curve: 0.641 (95\% Cl: $0.585-0.697)$. The sensitivity and specificity of the point with highest accuracy were $72.2 \%$ and $53.0 \%$, respectively. Abbreviations: $\mathrm{Cl}$, confidence interval; ROC, receiver operating curve.

independent prognostic factor of DFS, and primary tumor site and stage were also considered independent prognostic indicators of OS (left-sided vs right-sided: $\mathrm{HR}=0.238,95 \%$ confidence interval $[\mathrm{CI}]$ $=0.088-0.644$ ), $\mathrm{P}=0.005$ ) (Table 3). We next further analyzed the effects of NLN on survival in each subgroup of stage pN1 and pN2, left- and right-side disease, and found that NLN played a prognostic role in patients with right-side and stage $\mathrm{pN} 1$ disease compared with that in patients with stage $\mathrm{pN} 2$ and left-side disease. For patients with stage $\mathrm{pN} 1$, those with NLN $\leq 9$ showed an inferior 5-year OS rate $(71.1 \%$ vs $84.8 \% ; \mathrm{P}=0.009)$ and 3 -year DFS rate $(60.6 \%$ vs $80.5 \%$; $\mathrm{P}<0.001)$ than those with NLN $>9$. For right-side location, patients with NLN $\leq 9$ exhibited an inferior 5-year OS rate $(35.4 \%$ versus $77.1 \%, \mathrm{P}<$ $0.001)$ and 3-year DFS rate (34.4\% vs $78.9 \%, \mathrm{P}<0.001)$ than patients with NLN $>9$. For patients with stage $\mathrm{pN} 2$, those with NLN $\leq 9$ showed an inferior 3-year DFS rate $(51.2 \%$ vs $75.0 \% ; \mathrm{P}=0.012)$. For left-side location, patients with NLN $\leq 9$ exhibited an inferior 3 -year DFS rate $(62.9 \%$ versus $79.2 \%, \mathrm{P}=0.001)$. However, there was no association between the NLN and $\mathrm{OS}$ for patients with stage $\mathrm{pN} 2(\mathrm{P}=0.114)$ and left-side disease $(\mathrm{P}=0.086)$ (Figure 3$)$. As shown in table 1, patients with stage pN1 displayed a greater proportion of NLN $>9(68.4 \%$ vs $54.5 \%, \mathrm{P}=0.005)$ than those with stage $\mathrm{pN} 2$. There was no significant difference in neutrophil and lymphocyte infiltration, CRP and NLR between stage pN1and stage pN2. Regarding site, patients with right-side disease presented with a greater proportion of NLN $>9(79.7 \%$ vs $54.5 \%, \mathrm{P}<0.001)$ and CRP $>8.2(33.7 \%$ vs $17.1 \%, \mathrm{P}<0.001)$ than those with left-side disease. There was no significant difference in the neutrophil and lymphocyte infiltration and NLR between the right and left side.

As shown in the attached figures, in the low-grade neutrophil and lymphocyte infiltration group, patients with NLN > 9 exhibited meaningfully superior OS than those with NLN $\leq 9$. Nevertheless, there was no association of NLN and prognosis for patients with high-grade neutrophil and lymphocyte infiltration. In addition, NLN played a prognostic role for patients with CRP $\leq 8.2$ or NLR $>2.06$ compared with those with $\mathrm{CRP}>8.2$ or NLR $\leq 2.06$.

\section{Discussion}

The current study showed that increased NLN was significantly correlated with improved OS and DFS in stage III colon cancer. Subgroup analysis found that the prognostic value of NLN was limited to patients with right-side tumors and stage pN1 compared with those with right-side and stage $\mathrm{pN} 2$ disease.

The classification of NLN still lacks a uniform and reliable standard, with different studies using different stratification and statistical methods $[7,14]$. In the present study, the number of negative lymph nodes $\leq 9$ was found to be the most discriminatory threshold value among the different number of lymph node cutoffs according to ROC analysis.

Table 2. DFS rate and OS rate for stage III colon cancer stratified by the number of negative lymph nodes identified.

\begin{tabular}{lll}
\hline Negative Lymph Nodes & $\leq 9$ & $>9$ \\
\hline 2- DFS (\%) & 63.9 & 82.8 \\
3-DFS (\%) & 56.8 & 79.1 \\
5-OS (\%) & 68.6 & 82.7 \\
\hline
\end{tabular}

Abbreviations:

OS, overall survival; DFS, disease-free survival.

Table 3. Results of multivariate analyses of prognostic factors for DFS and OS.

\begin{tabular}{lllll}
\hline Parameter & DFS & \multicolumn{2}{l}{ OS } \\
\hline & HR $(95 \%$ CI $)$ & p-value & HR $(95 \%$ CI $)$ & p-value \\
\hline Primary tumor site & - & - & $0.238(0.088-0.644)$ & $\mathbf{0 . 0 0 5}$ \\
Stage (7th AJCC) & $0.931(0.510-1.698)$ & 0.851 & $0.340(0.139-0.831)$ & $\mathbf{0 . 0 1 8}$ \\
Negative lymph nodes & $0.309(0.187-0.509)$ & $<0.001$ & $0.204(0.073-0.567)$ & $\mathbf{0 . 0 0 2}$ \\
Ly invasive group & $0.242(0.090-0.651)$ & $\mathbf{0 . 0 0 5}$ & $0.132(0.016-1.067)$ & 0.058 \\
\hline Abbreviations: Ly, lymphocyte.
\end{tabular}

Abbreviations: Ly, lymphocyte. 

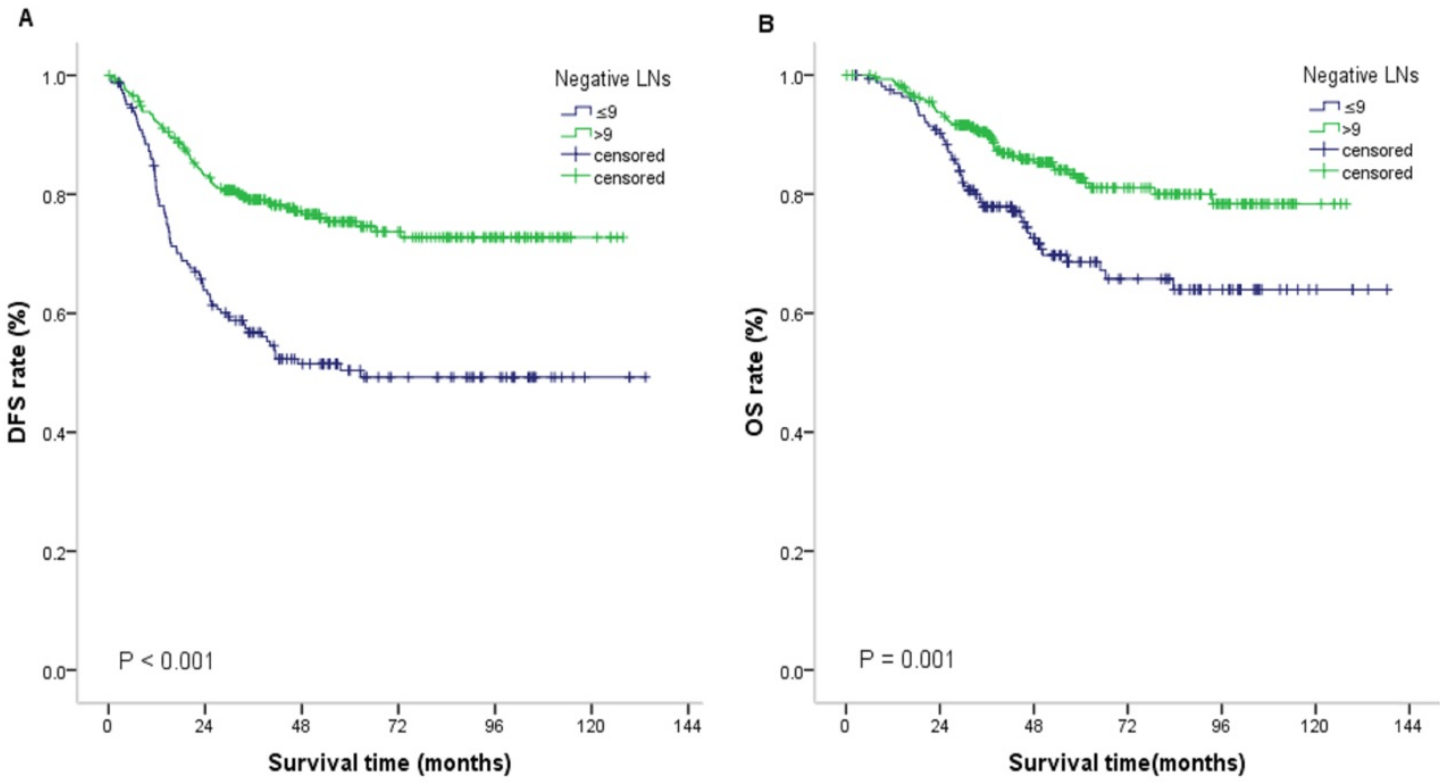

Figure 2. Disease-free survival (DFS) and Overall survival (OS) for patients with III stage colon cancer. Kaplan-Meier plots of DFS (A) and OS (B) according to negative lymph node counts $(\leq 9$ vs $>9)$. Abbreviations: LNs, lymph node counts
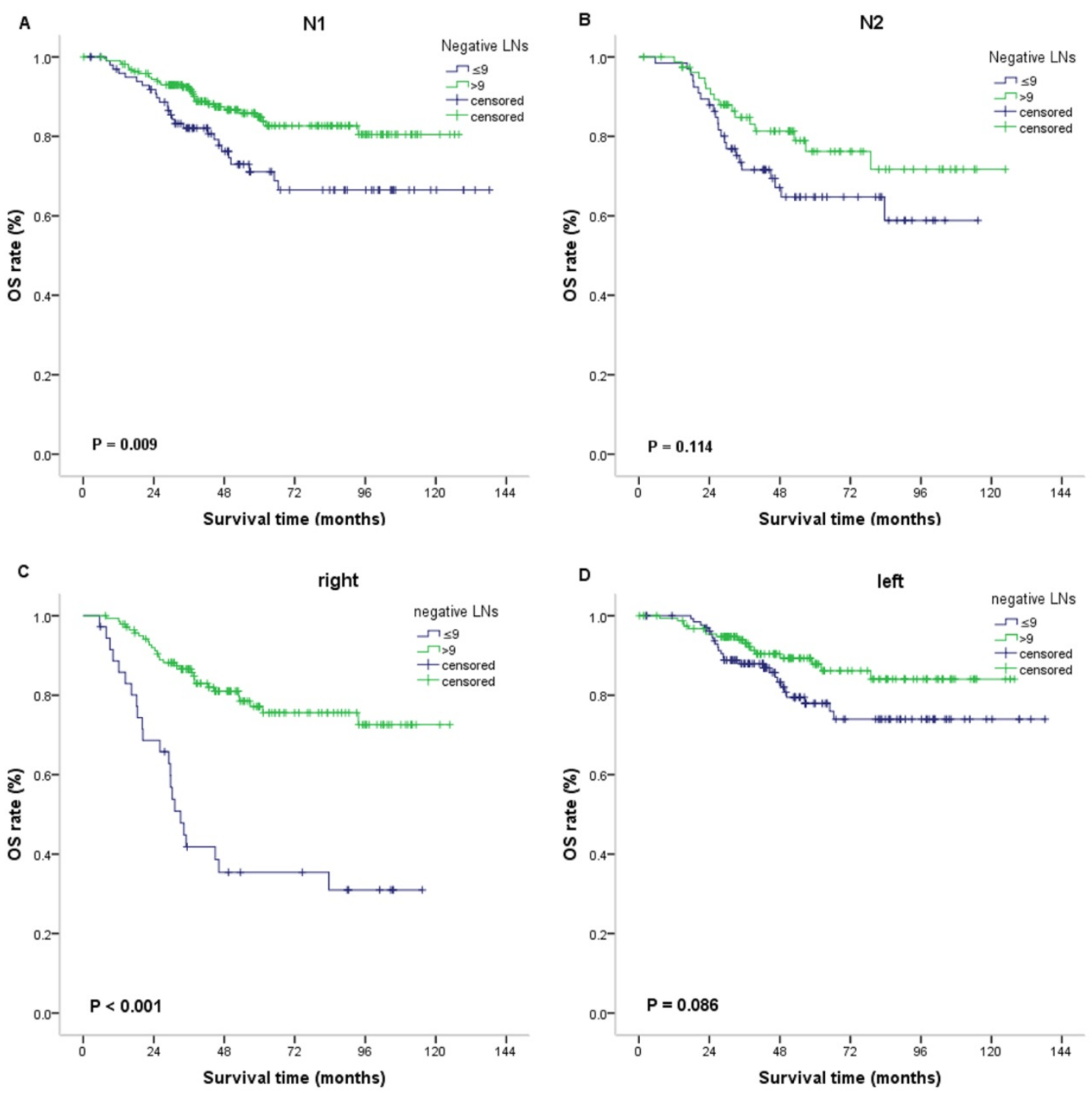

Figure 3. OS according to negative lymph node counts ( $\leq 9 \mathrm{vs}>9$ ) in the subgroups. Kaplan-Meier plots of OS in the subgroups of N1 (A) and N2 (B). Kaplan-Meier plots of OS in the subgroups of right colon cancer (C) and left colon cancer (D). 
Although the role of NLN in the prognosis of colon cancer has been gradually acknowledged by clinicians and pathologists, the mechanisms underlying the relationship between increased NLN and long-term survival have not been amply elucidated. It is likely that factors associated with lymph node harvest are crucial for the survival advantage. For example, the extent and quality of radical surgical resection can have an impact on the node harvest [15]. The number of regional lymph nodes retrieved from a surgical specimen also varies with the age of the patient, gender, and primary tumor location or tumor grade [4,16-18]. The NLN and number of total lymph nodes are positively related in general; thus, NLN is also affected by these factors correspondingly. More importantly, it was suggested that lymph nodes in patients with a strong anti-cancer immune response are easier to be discovered; therefore, a higher NLN in patients indicates a strong anti-cancer immune response to a tumor, and such patients show an improved survival [19]. By this theory, the NLN would be a marker of underlying tumor-host interactions, which may have an independent effect on survival. Another possibility is that the underlying tumor biology affects the lymph node yield and prognosis in parallel. For instance, MSI and wild-type KRAS/BRAF have been associated with both improved prognosis and increased lymph node retrieval, which we will improve in future research [20,21], although this information is missing for various reasons in our study.

Is it possible that the role of NLN in the prognosis is affected by an uneven distribution of clinicopathological features? As described in the "Results", patients in the NLN>9 group presented with a greater proportion of right-side colon cancer and stage $\mathrm{pN} 1$ disease than patients in the NLN $\leq 9$ group. In this study, we observed that survival was inferior for right-side compared with left-side colon cancer patients, displaying the opposite role in survival compared with NLN. It was shown that stage pN1 correlated with improved prognosis, showing an alternate effect as NLN. Thus, we can rule out the impact on the prognosis caused by an uneven distribution of the primary tumor site but not that cause by stage $\mathrm{pN} 1$ disease.

There was no association between the number of negative lymph nodes identified and overall survival for patients with stage $\mathrm{pN} 2$ disease. The possible reasons and potential mechanisms as follows. As previously mentioned, a strong immunologic reaction against the tumor could result in local lymph node hyperplasia with enlargement and enhanced lymph node detectability [19]. The greater the number of negative lymph nodes, the better the immune function of the body. Conversely, the greater the number of positive lymph nodes, the worse the immune function of the body. For patients with a higher $\mathrm{N}$ stage ( $\mathrm{pN} 2)$, the tumor invasion ability is more powerful than body immunity in the fight against the development of cancer. Therefore, the $\mathrm{NLN}$, as an indicator of the immune status of the reaction, may not play an important role in prognosis to a relative degree. In addtion, patients with pN2 have relatively high NLR values (the neutrophil lymphocyte ratio), which denotes that the balance is tipped in favor of tumor inflammatory response and is associated with poor oncologic outcome. In other words, inflammation plays a more important role than immune function at this time.

We found that survival was inferior for right-side compare with that for left-side colon cancer patients. Multivariable analyses confirmed right-side location as a negative prognostic variable, a finding that is inconsistent with those of several other studie $[22,23]$. Safiya Karim and others found no strong association between disease laterality and outcome of early-stage resected colon cancer. In the subgroup analysis, the number of negative lymph nodes did not significantly predict the prognosis in patients with left-side colon cancer. Several potential interpretations have been suggested. First, the overall prognosis for left-side disease was excellent, and thus our study, although large, may have insufficient power. Second, The patients with right-side has more negative lymph nodes, which means that the immune is stronger, while left-side disease is likely considered to correlate with less immune activation than right-side disease, thus, the role of immunity is not great. The patients with left-side have lower levels of neutrophils and CRP, indicating that left-side disease is weaker in both inflammatory and immune responses than right-side disease.

We found no association between the NLN and survival outcome for patients in each subgroup of high-grade neutrophil and lymphocyte infiltration. Lymphocytes play an important role in host defenses against tumors because they inhibit the proliferation and invasion of tumor cells via the induction of cytotoxic cell death and cytokine production. Previous data have shown that neutrophil infiltration plays an important role in stimulating tumor growth, angiogenesis and metastasis [24,25]. In this study, more lymphocyte infiltration was significantly positively correlated with long survival; thus, the analysis of negative lymph nodes regarding prognosis in the subgroup is relatively meaningless. As a marker of systemic inflammation, the pre-treatment NLR and CRP have been recognized as prognostic factors in colon cancer. Negative lymph nodes in the high CRP 
subgroup did not show prognostic significance; however, those in the low CRP group were significant, likely because the low level of CRP indicates inflammation is more powerful than body immunity in the fight against tumors, causing NLN to be associated with the immune response out of action. However, NLN in the subgroup of low NLR did not show prognostic significance, contrary to the above theory. These phenomena may be related to the lack of our sample size; more importantly, other mechanisms involved are unclear. Here, it must be mentioned that there was a significant difference in the DFS and OS between the NLN $\leq 9$ and $>9$ groups in only stage IIIB disease compared with that in stage IIIA and IIIC disease. The patients with stage IIIB accounted for $79.8 \%$ of the total number of patients, while the number of IIIA and IIIC patients only accounted for $20.2 \%$, making it difficult to draw meaningful results.

Our study should be interpreted in the context of certain methodological limitations. The study does not include information regarding various known prognostic factors in colon cancer, such as smoking status, performance status or diet. Therefore, residual confounding is a major limitation in this study. In addition, we could not collect the molecular tumor subtype, which likely plays a role in the prognosis. Additionally, the present study is limited due to its retrospective, single-center design. The major strengths of the study are that we first evaluated the effects of NLN on survival in each subgroup of patients with stage pN1 and pN2, left- and right-side disease.

\section{Conclusion}

Our study demonstrated that the negative lymph node count can function as an independent indicator of the survival outcome in stage III colon cancer, only for patients with right-side or stage $\mathrm{pN} 1$ disease. The relationship may be immune-related, and the specific mechanism remains uncertain. Future research with a large number of cases that can incorporate molecular and genetic elements are needed.

\section{Acknowledgements}

This study was supported by grants from the Natural Science Foundation of Guangdong, China (2015A030313010), Science and Technology Program of Guangzhou, China (1563000305) and National Natural Science Foundation of China (81572409).

\section{RDDN of the dataset}

RDDA2018000727.

\section{Competing Interests}

The authors have declared that no competing interest exists.

\section{References}

1. Cohen AM. Surgical considerations in patients with cancer of the colon and rectum. Seminars in oncology. 1991; 18: 381-7.

2. West NP, Hohenberger W, Weber K, Perrakis A, Finan PJ \& Quirke P. Complete mesocolic excision with central vascular ligation produces an oncologically superior specimen compared with standard surgery for carcinoma of the colon. Journal of clinical oncology : official journal of the American Society of Clinical Oncology. 2010; 28: 272-8.

3. Chau I \& Cunningham D. Adjuvant therapy in colon cancer--what, when and how? Annals of oncology : official journal of the European Society for Medical Oncology. 2006; 17: 1347-59.

4. Le Voyer TE, Sigurdson ER, Hanlon AL, Mayer RJ, Macdonald JS, Catalano PJ, et al. Colon cancer survival is associated with increasing number of lymph nodes analyzed: a secondary survey of intergroup trial INT-0089. Journal of clinical oncology : official journal of the American Society of Clinical Oncology. 2003; 21: 2912-9.

5. Fang HY, Yang H, He ZS, Zhao H, Fu ZM, Zhou FX, et al. Log odds of positive lymph nodes is superior to the number- and ratio-based lymph node classification systems for colorectal cancer patients undergoing curative (R0) resection. Molecular and clinical oncology. 2017; 6: 782-8.

6. Berger AC, Sigurdson ER, LeVoyer T, Hanlon A, Mayer RJ, Macdonald JS, et al. Colon cancer survival is associated with decreasing ratio of metastatic to examined lymph nodes. Journal of clinical oncology : official journal of the American Society of Clinical Oncology. 2005; 23: 8706-12.

7. Johnson PM, Porter GA, Ricciardi R \& Baxter NN. Increasing negative lymph node count is independently associated with improved long-term survival in stage IIIB and IIIC colon cancer. Journal of clinical oncology : official journal of the American Society of Clinical Oncology. 2006; 24: 3570-5.

8. Schinzari G, Rossi E, Cassano A, Dadduzio V, Quirino M, Pagliara M, et al. Cisplatin, dacarbazine and vinblastine as first line chemotherapy for liver metastatic uveal melanoma in the era of immunotherapy: a single institution phase II study. Melanoma research. 2017; 27: 591-5.

9. Wu SG, Wang Y, Zhou J, Sun JY, Li FY, Lin HX, et al. Number of negative lymph nodes should be considered for incorporation into staging for breast cancer. American journal of cancer research. 2015; 5: 844-53.

10. Chen Y, Zhang L, Tian J, Ren X \& Hao Q. Combining the negative lymph nodes count with the ratio of positive and removed lymph nodes can better predict the postoperative survival in cervical cancer patients. Cancer cell international. 2013; 13: 6

11. Kiyota N, Hasegawa Y, Takahashi S, Yokota T, Yen CJ, Iwae S, et al. A randomized, open-label, Phase III clinical trial of nivolumab vs. therapy of investigator's choice in recurrent squamous cell carcinoma of the head and neck: A subanalysis of Asian patients versus the global population in checkmate 141. Oral oncology. 2017; 73: 138-46.

12. Park JH, Powell AG, Roxburgh CS, Horgan PG, McMillan DC \& Edwards J. Mismatch repair status in patients with primary operable colorectal cancer: associations with the local and systemic tumour environment. British journal of cancer. 2016; 114: 562-70.

13. Klintrup K, Makinen JM, Kauppila S, Vare PO, Melkko J, Tuominen H, et al. Inflammation and prognosis in colorectal cancer. European journal of cancer (Oxford, England : 1990). 2005; 41: 2645-54.

14. Ogino S, Nosho K, Irahara N, Shima K, Baba Y, Kirkner GJ, et al. Negative lymph node count is associated with survival of colorectal cancer patients, independent of tumoral molecular alterations and lymphocytic reaction. The American journal of gastroenterology. 2010; 105: 420-33.

15. Wong SL, Ji H, Hollenbeck BK, Morris AM, Baser O \& Birkmeyer JD. Hospital lymph node examination rates and survival after resection for colon cancer. Jama. 2007; 298: 2149-54.

16. Bilimoria KY, Palis B, Stewart AK, Bentrem DJ, Freel AC, Sigurdson ER, et al. Impact of tumor location on nodal evaluation for colon cancer. Diseases of the colon and rectum. 2008; 51: 154-61.

17. Nedrebo BS, Soreide K, Nesbakken A, Eriksen MT, Soreide JA \& Korner H. Risk factors associated with poor lymph node harvest after colon cancer surgery in a national cohort. Colorectal disease : the official journal of the Association of Coloproctology of Great Britain and Ireland. 2013; 15: e301-8.

18. Sarli L, Bader G, Iusco D, Salvemini C, Mauro DD, Mazzeo A, et al. Number of lymph nodes examined and prognosis of TNM stage II colorectal cancer. European journal of cancer (Oxford, England : 1990). 2005; 41: 272-9.

19. Wong SL. Lymph node evaluation in colon cancer: assessing the link between quality indicators and quality. Jama. 2011; 306: 1139-41.

20. Belt EJ, te Velde EA, Krijgsman O, Brosens RP, Tijssen M, van Essen HF, et al. High lymph node yield is related to microsatellite instability in colon cancer. Annals of surgical oncology. 2012; 19: 1222-30.

21. Berg M, Guriby M, Nordgard O, Nedrebo BS, Ahlquist TC, Smaaland R, et al. Influence of microsatellite instability and KRAS and BRAF mutations on lymph node harvest in stage I-III colon cancers. Molecular medicine (Cambridge, Mass). 2013; 19: 286-93. 
22. Karim S, Brennan K, Nanji S, Berry SR \& Booth CM. Association Between Prognosis and Tumor Laterality in Early-Stage Colon Cancer. JAMA oncology. 2017; 3: 1386-92.

23. Chang GJ \& Gonen M. Prognostic and Predictive Ability of Tumor Sidedness: Another Vexing Difference Between Localized and Advanced Colon Cancer. JAMA oncology. 2017; 3: 1314-5.

24. Jablonska I, Leschner S, Westphal K, Lienenklaus S \& Weiss S. Neutrophils responsive to endogenous IFN-beta regulate tumor angiogenesis and growth in a mouse tumor model. The Journal of clinical investigation. 2010; 120: 1151-64.

25. Nozawa H, Chiu C \& Hanahan D. Infiltrating neutrophils mediate the initial angiogenic switch in a mouse model of multistage carcinogenesis. Proceedings of the National Academy of Sciences of the United States of America. 2006; 103: $12493-8$. 